\title{
Novel Thione-Thiol Rearrangement of Dithiocarbonic Acid 0-(2,2,6,6-Tetra- methylpiperidin-1-yl) Ester in the Context of Controlled Radical Polymerization
}

\author{
Peter Nesvadba*a, Lucienne Bugnon ${ }^{a}$, and Trixie Wagner ${ }^{\mathrm{b}}$
}

\section{Dedicated to Professor Daniel Belluš on the occasion of his 70th birthday}

\begin{abstract}
Addition of the lithium salt of N-hydroxy-2,2,6,6-tetramethylpiperidine 10 to carbon disulfide and subsequent methylation affords the rearranged dithiocarbonic acid S-methyl-S'-(2,2,6,6-tetramethylpiperidin-1-yl)ester 9 rather than the expected xanthate ester S-methyl-O'-(2,2,6,6-tetramethylpiperidin-1-yl)-ester 8. n-Butylacrylate could be polymerized with the novel compound $\mathbf{9}$ even though the polymerization was not controlled.
\end{abstract}

Keywords: Acyloxyamines · Alkoxyamines · Controlled/living radical polymerization ·

Newman-Kwart rearrangement $\cdot$ Nitroxides $\cdot$ Thione-thiol rearrangement $\cdot$ Thionitroxides

\section{Introduction}

Ciba has a long-standing tradition and leadership in the chemistry of sterically hindered amines. These derivatives of 2,2,6,6-tetramethylpiperidine (TMP) are used as stabilizers to protect synthetic polymers against degradation induced by light and heat. The related nitroxide radicals inhibit the lignin photo-oxidation and thus ensure that wood keeps its natural color and attractive appearance. Recently, the TMP-N-alkoxyamines emerged as environmentally friendly halogen-free flame retardants for plastics. ${ }^{[1]}$ The next opportunity to further develop the TMP chemistry and expand it beyond the aforementioned applications appeared with invention of controlled/living radical polymerization $(\mathrm{CRP})^{[2,3]}$ some 20 years ago. From the different CRP techniques known, the nitroxide-mediated controlled/living radical polymerization (NMP) ${ }^{[4]}$ was the most attractive for Ciba to pursue because the initiators/regulators for NMP are N-alkoxyamines and nitroxides which belong to the Ciba's core competence.
${ }^{\star}$ Correspondence: PD Dr. P. Nesvadba ${ }^{a}$

Tel.: +41616362412

Fax: +41616362332

E-mail: peter.nesvadba@basf.com

${ }^{a}$ Ciba Inc. (now part of BASF)

Performance Chemicals Research, R-1059.6.05

Klybeckstr. 141

$\mathrm{CH}-4002$ Basel

bNovartis Institutes for BioMedical Research

Lichtstrasse 35

$\mathrm{CH}-4056$ Basel
The intensive research work on NMP at Ciba resulted in the development of novel highly efficient alkoxamines and nitroxides $^{[5-8]}$ and Ciba was the first company worldwide to realize NMP on industrial scale. Several new products, e.g. pigment dispersants, prepared by NMP have already been introduced to the market. ${ }^{[1]}$ The journey towards industrially feasible NMP was not always foreseeable but also accompanied with unexpected results. One example of such a serendipity observation is the topic of this short article.

Thione to thiol rearrangements are well-known. For example O,S-dialkyl xanthates 1 rearrange thermally[9,10] or under base catalysis ${ }^{[11,12]}$ to S,S-dialkyl dithiocarbonates 1a (Scheme 1). Upon heating, ketoxime O-(S-methyl xanthates) 2 rearrange ${ }^{[13]}$ to $\mathrm{S}$-imino-S'-methyl-dithiocarbonates $\mathbf{2 a}$ and N,N-dimethylthiocarbamoyl-N'-methylbenzohydroxamates 3 are transformed[14] already at room tem- perature slowly into N,N-dimethylcarbamoyl-N'-methylbenzothiohydroxamates 3a. Synthetically important is the thermal Newman-Kwart rearrangement ${ }^{[15,16]}$ of $\mathrm{O}$ arylthio carbamates $\mathbf{4}$ into S-arylthiocarbamates 4a which enables the transformation of phenols ArOH into the corresponding thiophenols ArSH. Depending on the actual substrate, both ionic ${ }^{[11,12,16]}$ or free radical[ ${ }^{[13,14]}$ mechanisms were postulated for these rearrangements.

During our work on nitroxide-mediated controlled/living radical polymerization (NMP) ${ }^{[4]}$ we have developed various classes of novel $\mathrm{N}$-alkoxyamines ${ }^{[5-8]}$ which work as efficient initiators/regulators for NMP. In the same context we observed that the structurally closely related N-acyloxy-2,2,6,6-tetramethylpiperidines do not induce NMP but rather behave as classical free radical initiators. ${ }^{[17]}$ The principle of NMP is based on the reversible, thermally induced homolysis of the weak (depending on the actual struc-<smiles>[R]OC(=S)S[R]</smiles><smiles>[R]SC(=O)S[R]</smiles>

1
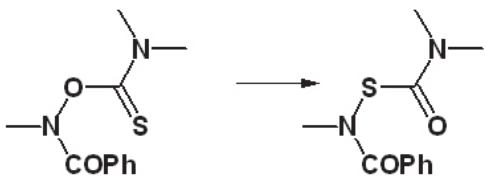

$3 a$<smiles>[R]C([R])=NOC(=S)SC</smiles>
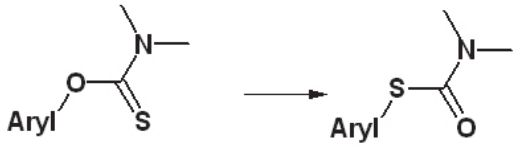

$4 a$

Scheme 1. Examples of thione-thiol rearrangements. 
ture $c a .115-135 \mathrm{~kJ} / \mathrm{mol})^{[6,18]} \mathrm{NO}-\mathrm{C}$ bond of alkoxyamines affording persistent nitroxide radicals and reactive $\mathrm{C}$-centered radicals, as exemplified on 2,2,6,6-tetramethyl-1-(1-phenyl-ethoxy)-piperidine $\mathbf{5}$ (Scheme 2). Our density functional (DFT) calculations (Scheme 2 and details in the experimental part) of bond dissociation enthalpies (BDE) of the strategic NO-C and $\mathrm{N}-\mathrm{OC}$ bonds in $\mathbf{5}$ and in the model compounds, N-acetyloxy-2,2,6,6-tetramethylpiperidine $\mathbf{6}$ and $\mathrm{N}$-methoxycarbonyloxy-2,2,6,6-tetramethylpiperidine 7 support this observation. Indeed, the calculation predicts for the $\mathrm{NO}-\mathrm{C}$ bond in the alkoxyamine 5 a BDE of $122 \mathrm{~kJ} / \mathrm{mol}$ which is in excellent agreement with the experimental value ${ }^{[18]}(119 \mathrm{~kJ} / \mathrm{mol})$ and which is less than the BDE of the $\mathrm{N}-\mathrm{OC}$ bond $(200 \mathrm{~kJ} / \mathrm{mol})$. The situation is just opposite in the $\mathrm{N}$-acyloxyamines $\mathbf{6}$ and 7 which suggests that homolysis of these compounds affords two reactive radicals, namely 2,2,6,6-tetramethylpiperidinyl and acetyloxy or methoxycarbonyloxy, respectively. These radicals would directly or indirectly initiate the uncontrolled polymerization. The same calculations predict even weaker $\mathrm{N}-\mathrm{OC}$ bond in the unknown N-methylthiothiocarbonyloxy-2,2,6,6tetramethylpiperidine 8 . On the other hand the dithiolcarbonate isomer 9 should homolyse along the NS-C bond to afford 2,2,6,6-tetramethylpiperidinyl-thionitroxide and methylthiocarbonyl radicals. The low calculated BDE value $(114 \mathrm{~kJ} / \mathrm{mol})$ of the $\mathrm{N}-\mathrm{OC}$ bond in $\mathbf{8}$ led us to speculate that this compound may behave as a free radical initiator working at lower temperature than derivatives of $\mathbf{6}$ or $\mathbf{7}$ and hence prompted us to attempt its synthesis. The obvious strategy appeared to be the addition of the anion of N-hydroxy-2,2,6,6-tetramethylpiperidine $\mathbf{1 0}$ (Scheme 3) to carbon disulfide followed by methylation. Surprisingly, we have found that addition of the lithium salt of $\mathbf{1 0}$ to $\mathrm{CS}_{2}$ in THF and subsequent treatment with methyl iodide affords the dithiocarbonic acid S-methyl-S'-(2,2,6,6tetramethylpiperidin-1-yl) ester 9 in $77 \%$ yield instead of the expected S-methyl-O(2,2,6,6-tetramethyl-piperidin-1-yl) ester 8. This unexpected reaction is thus a new example of a thione-thiol rearrangement.

\section{Experimental}

Solvents and reagents are commercially available and were used as received.

${ }^{1} \mathrm{H}$ and ${ }^{13} \mathrm{C}$ NMR spectra were recorded on a Bruker Avance 300 spectrometer (300 MHz for ${ }^{1} \mathrm{H}$ and $75.47 \mathrm{MHz}$ for ${ }^{13} \mathrm{C}$ ). Chemical shifts $(\delta)$ are given in ppm downfield from internal $\mathrm{Me}_{4} \mathrm{Si}$ standard, J values are in Hz. IR spectra were taken on Nicolet Magna-IR 750 spectrometer in ATR mode,

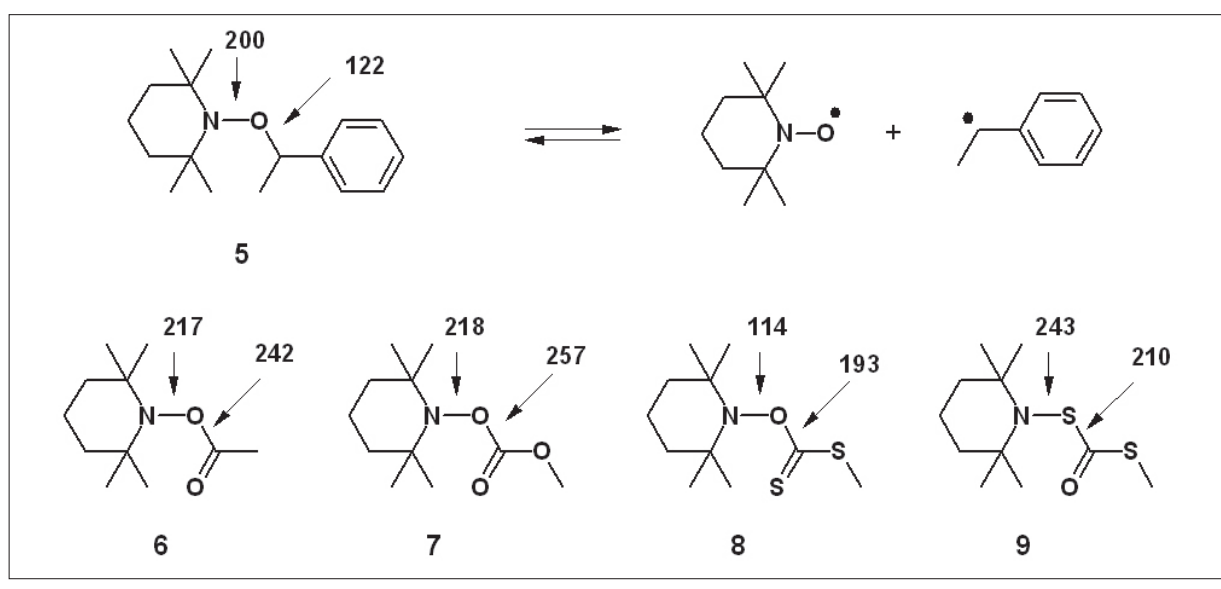

Scheme 2. Calculated bond dissociation enthalpies in the compounds $5-9(\mathrm{~kJ} / \mathrm{mol}, 298 \mathrm{~K}, 1 \mathrm{~atm}$, see introduction).

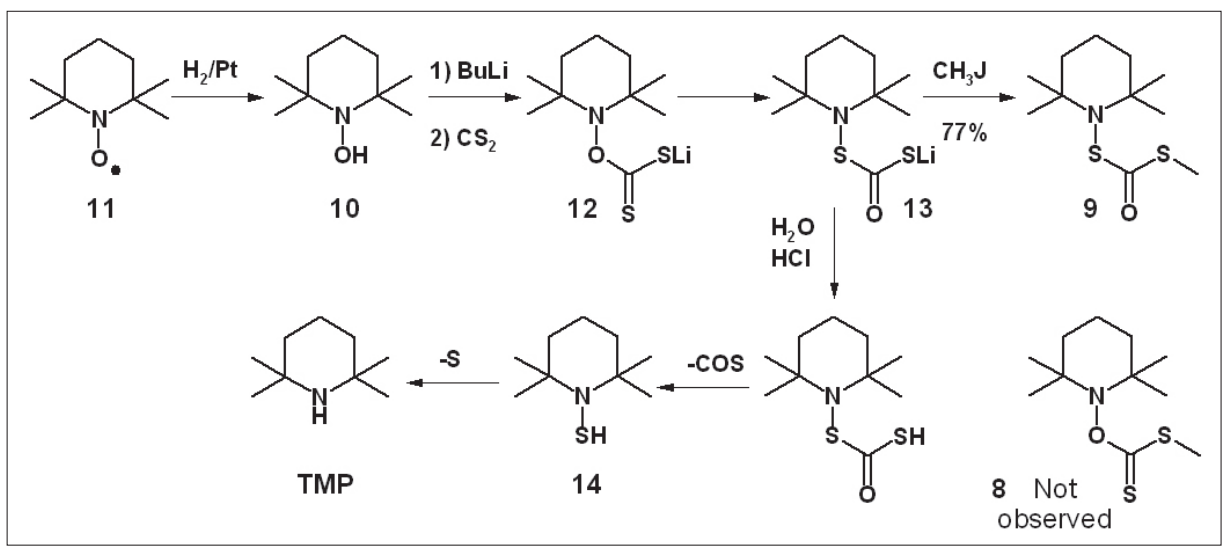

Scheme 3. Synthesis of the rearranged compound $\mathbf{9}$.

MS spectra on Finnigan SSQ 710 apparatus. Elemental analyses were obtained from the Ciba Service Center Elemental Analysis.

The GPC analysis of the polymer was performed on a set of 2 PL-Gel $300 \times 7.5 \mathrm{~mm}(5 \mu \mathrm{m})$ mixed $\mathrm{C}$ columns + 1 PL-Gel guard column using a Spark Holland Basic Marathon sampler and a Flux RHEOS 4000 pump with inline ERC 1215 $\alpha$ degasser, coupled with ERC 7515A refractometer and ERC7217 UV detector. The columns temperature was $40^{\circ} \mathrm{C}$, the detector temperature $30^{\circ} \mathrm{C}$. Tetrahydrofuran was used as eluent ( $1 \mathrm{ml} / \mathrm{min})$. Polystyrene standards were used for the calibration.

The X-ray structure determination was carried out at Novartis Institutes for BioMedical Research, Basel, Switzerland. [19]

The quantum chemical calculations were conducted with Spartan 06 (Wavefunction, Inc., USA) and Gaussian 03 (Gaussian Inc., USA) suites of programs. The ROB3P86/6-311G(2d,2p)//B3LYP/6$31 \mathrm{Gd}$ method, which was used to calculate the bond dissociation enthalpies (including zero point energy and corrections to $298 \mathrm{~K}$, $1 \mathrm{~atm}$ ), is a slight modification (geometry optimization was performed at B3LYP/6$31 \mathrm{Gd}$ instead of B3P86/6-31Gd level) of the well-established procedure ${ }^{[20]}$ of DiLabio and coworkers. The relative energies of 8 and 9 were calculated RB3LYP/6$311+\mathrm{G}(2 \mathrm{~d}, \mathrm{p}) / / \mathrm{RB} 3 \mathrm{LYP} / 6-31 \mathrm{G}(\mathrm{d})$ and RMP2-fc/6-311+G(2d,p)//RMP2-fc/6$31 \mathrm{G}(\mathrm{d})$ levels. The minima character of all optimized species was confirmed by frequency calculations at the same level, the vibrational frequencies were scaled by the appropriate scaling factors. ${ }^{[21]}$

\subsection{Dithiocarbonic Acid S-Methyl Ester S-(2,2,6,6-tetramethyl-piperi- din-1-yl) Ester (9)}

A solution of 2,2,6,6-tetramethylpiperidine-1-oxyl $(\mathbf{1 1}, 23.43 \mathrm{~g}, 0.15 \mathrm{~mol}$, Fluka $>96 \%)$ in THF $(100 \mathrm{ml}$, dried over molecular sieve) and platinum on charcoal (5\% loading) catalyst $(450 \mathrm{mg}$ ) was hydrogenated at room temperature and 2 bar pressure until the hydrogen uptake ceased (45 min). The mixture was then rapidly filtered into a 11 three-necked flask filled with argon, the catalyst was washed with THF $(150 \mathrm{ml})$ and the slightly yellow filtrate was cooled to $0{ }^{\circ} \mathrm{C}$. Thereafter, $1.6 \mathrm{M}$ solution of n-butyl lithium in hexane (100 ml, 0.16 mol, Fluka) was added during 45 min and at $0-2{ }^{\circ} \mathrm{C}$ and the mixture was stirred for another $30 \mathrm{~min}$ at $0{ }^{\circ} \mathrm{C}$ under argon. Carbon disulfide (12.56 g, 0.165 mol, Fluka >99\%) was then added at 0-2 ${ }^{\circ} \mathrm{C}$ during $20 \mathrm{~min}$. The solution, which 
turned from slightly yellow into orange, was stirred at $0{ }^{\circ} \mathrm{C}$ for $75 \mathrm{~min}$. Methyl iodide (23.42 g, 0.165 mol, Fluka >99\%) was then added during 45 min while keeping the temperature at $0-2{ }^{\circ} \mathrm{C}$ and the solution was stirred for additional hour at $0{ }^{\circ} \mathrm{C}$. The resulting orange-yellow solution was then evaporated on a rotary evaporator and water $(100 \mathrm{ml})$ and methyl-t-butyl ether $(150$ $\mathrm{ml}$ ) was added to the residue. The organic phase was separated, the aqueous layer was diluted with $25 \% \mathrm{NaCl}$ solution (50 $\mathrm{ml})$ and extracted with methyl-t-butyl ether $(2 \times 50 \mathrm{ml})$. The combined organic layers were washed with $25 \% \mathrm{NaCl}$ solution $(2 \times$ $40 \mathrm{ml}$ ), dried over $\mathrm{MgSO}_{4}$ and evaporated. The solid, slightly yellow residue (36.4 g) was dissolved in toluene $(50 \mathrm{ml})$ and let crystallize at $-20{ }^{\circ} \mathrm{C}$ to afford $28.7 \mathrm{~g}$ $(77.3 \%)$ of 9 as almost white, malodorous crystals, mp. $81-83{ }^{\circ} \mathrm{C}$.

Anal. calcd. for $\mathrm{C}_{11} \mathrm{H}_{21} \mathrm{NOS}_{2}$ (247.42): C, 53.40; H, 8.56; N, 5.66; S, 25.92. Found: C, 53.46; H, 8.35; N, 5.57; S, 25.94. MS(ESI): $\left.\mathrm{m} / \mathrm{z}(\%)=248.2(100)[\mathrm{M}+\mathrm{H}]^{+}\right), 142.3(60)$. IR, cm ${ }^{-1}: 1618(\mathrm{C}=\mathrm{O}) .{ }^{1} \mathrm{H}-\mathrm{NMR}(300 \mathrm{MHz}$, $\left.\mathrm{CDCl}_{3}\right): \delta=2.33\left(\mathrm{~s}, 3 \mathrm{H}, \mathrm{SCH}_{3}\right), 1.8-1.4(\mathrm{~m}$, $\left.6 \mathrm{H}, 3 \times \mathrm{CH}_{2}\right), 1.34\left(\mathrm{~s}, 6 \mathrm{H}, 2 \times \mathrm{CH}_{3}\right), 1.17$ (s, $\left.6 \mathrm{H}, 2 \times \mathrm{CH}_{3}\right) .{ }^{13} \mathrm{C}-\mathrm{NMR}(75.38 \mathrm{MHz}$, $\left.\mathrm{CDCl}_{3}\right): \delta=201.2(\mathrm{CO}), 60.1,40.5,32.2$, $25.3,17.3,12.4$. Crystals for X-ray analysis were grown from acetonitrile.

\subsection{Bulk Polymerization of n-Butylacrylate with (9)}

A magnetically stirred solution of 9 (0.247 g, $1 \mathrm{mmol})$ in n-butyl acrylate $(12.82 \mathrm{~g}, 10 \mathrm{mmol})$ in a $50 \mathrm{ml}$ round flask was degassed by three vacuum/argon cycles and was then heated at $140{ }^{\circ} \mathrm{C}$ for 90 min to afford colorless, very viscous polymer. Integration of the ${ }^{1} \mathrm{H}-\mathrm{NMR}\left(\mathrm{CDCl}_{3}\right)$ signals of the $\mathrm{OCH}_{2}$ groups of the unreacted monomer $(4.16 \mathrm{ppm})$ and polymer (4.0 ppm) indicated 92\% conversion. GPC: $M_{n}=67291, M_{w}=580861, M_{w} / M_{n}=8.6$.

\section{Results and Discussion}

Reports on reactions of N,N-dialkyl hydroxylamines with carbon disulfide are very scarce. Haase and Wolffenstein observed deoxygenation of diethyl- or dibenzyl hydroxylamine upon heating with $\mathrm{CS}_{2}$, however the structure of the products was not conclusively elucidated.[22] Recently, preparation of stable O-dialkylamino xanthates starting from diethyl- or benzylphenyl hydroxylamine and their use as agents for controlled/living radical polymerization was described ${ }^{[23]}$ in the patent literature, however, no analytical data were provided for these compounds.

Our attempted synthesis of $\mathbf{8}$ is depicted in Scheme 3. Catalytic hydrogenation of 2,2,6,6-tetramethylpiperidine-N-oxyl

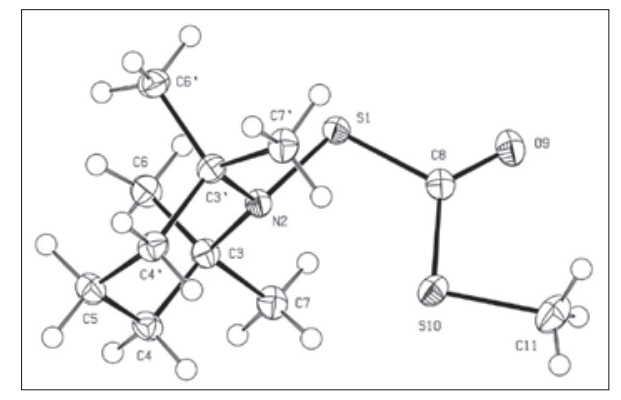

Fig. 1. X-ray structure of 9.

11 afforded in quantitative yield the hydroxylamine 10. Deprotonation of $\mathbf{1 0}$ with n-butyllithium in THF followed by subsequent addition of $\mathrm{CS}_{2}$ and methyl iodide yielded the rearranged compound 9 in $77 \%$ isolated yield instead of the expected isomer $\mathbf{8}$. The TLC of the crude reaction mixture before isolation showed that 9 is the only product formed. The structure of 9 has been confirmed by elemental analysis, mass spectrometry, ${ }^{1} \mathrm{H}$ and ${ }^{13} \mathrm{C}-\mathrm{NMR}$ and IR-spectroscopy. Most striking is the presence of the strong $>\mathrm{C}=\mathrm{O}$ group absorption at $1618 \mathrm{~cm}^{-1}$, the $>\mathrm{C}=\mathrm{S}$ group of the isomer $\mathbf{8}$ would not absorb in this region. Moreover, the ${ }^{13} \mathrm{C}$-resonance at $201.2 \mathrm{ppm}$ is compatible with the structure 9 but not $\mathbf{8}$ whose $>\mathrm{C}=\mathrm{S}$ signal is expected between 230-240 ppm. The ultimate proof of the structure 9 was obtained by X-ray analysis of single crystals grown from acetonitrile (Fig. 1).

The detailed mechanism of this novel rearrangement is not clear yet. However, the observation that addition of one equivalent of aqueous $\mathrm{HCl}$ instead of methyl iodide affords 2,2,6,6-tetramethylpiperidine (TMP) and elemental sulfur instead of $\mathbf{1 0}$ and $\mathrm{CS}_{2}$ suggests that it is the Li-xanthogenate 12 which rearranges into the Lidithiocarbonate 13. The methylation of $\mathbf{1 3}$ then affords $\mathbf{9}$. The formation of TMP and sulfur via the mercapto amine $\mathbf{1 4}$ can be plausibly explained (Scheme 3). Decomposition of $\mathrm{N}$-mercapto amines to amines and sulfur is known. [24]

We suppose that the main contribution to the thermodynamic driving force of the novel rearrangement originates from the transformation of a relatively weak $\mathrm{C}=\mathrm{S}$ bond into a strong $\mathrm{C}=\mathrm{O}$ bond. Indeed, calculations with DFT and Moeller-Plesset theory (see Experimental) lead to the same result indicating that the ground state en$\operatorname{ergy}(298 \mathrm{~K}, 1 \mathrm{~atm})$ of 9 is $132.9 \mathrm{~kJ} / \mathrm{mol}$ resp. $132.5 \mathrm{~kJ} / \mathrm{mol}$ lower than that of $\mathbf{8}$.

The compound $\mathbf{9}$ is a protected thionitroxide, the novel rearrangement thus opens a new access to thionitroxide derivatives. ${ }^{[25]}$ Bricklebank and Pryke report$\mathrm{ed}^{[26]}$ that radical polymerization of styrene in the presence of 2,2,6,6-tetramethylpiperidyl-1-thiyl affords polystyrene with high polydispersity $\left(\mathrm{M}_{\mathrm{w}} / \mathrm{M}_{\mathrm{n}}=1.9-4\right)$ and is therefore not controlled/living.

We were able to polymerize bulk nbutylacrylate with $1 \mathrm{~mol} \%$ of 9 at $140{ }^{\circ} \mathrm{C}$. However, the corresponding polymer, obtained in $92 \%$ yield, was highly polydisperse $\left(M_{w} / M_{n}=8.6\right)$ which indicates an uncontrolled polymerization in this case too.

\section{Conclusions}

We have reported a novel thione-thiol rearrangement of dithiocarbonic acid O(2,2,6,6-tetramethylpiperidin-1-yl) ester affording the first example of the hitherto unknown thionitroxide derivatives. The new compound 9 is a radical initiator for the thermal polymerization n-butylacrylate even though the polymerization is not controlled/living.

Received: December 18, 2009

[1] W. Rutsch, M. A. Cech, Chimia 2007, 61, 33.

[2] W. A. Braunecker, K. Matyjaszewski, Progr. Polym. Sci. 2007, 32, 93.

[3] G. Moad, E. Rizzardo, S. H. Thang, Acc. Chem. Res. 2008, 41, 1133.

[4] D. H. Solomon, J. Polym. Sci., Part A: Polym. Chem. 2005, 43, 5748.

[5] P. Nesvadba, L. Bugnon, R. Sift, J. Polym. Sci., Part A: Polym. Chem. 2004, 42, 3332.

[6] H. Fischer, A. Kramer, S. R. A. Marque, P. Nesvadba, Macromolecules 2005, 38, 9974.

[7] P. Nesvadba, Chimia 2006, 60, 832.

[8] S. Miele, P. Nesvadba, A. Studer, Macromolecules 2009, 42, 2419.

[9] T. Taguchi, M. Nakao, Tetrahedron 1962, 18, 245.

[10] T. Taguchi, Y. Kawazoe, M. Nakao, Tetrahedron Lett. 1963, 4, 131.

[11] H. Nakagawa, M. Eto, K. Harano, Tetrahedron 1998, 54,9333

[12] H. Nakagawa, M. Eto, K. Harano, Heterocycles 1999, $51,51$.

[13] F. Gagosz, S. Z. Zard, Synlett 1999, 1978.

[14] W. B. Ankers, R. F. Hudson, A. J. Lawson, J. Chem. Soc., Perkin Trans. 2 1974, 1826.

[15] C. Zonta, O. De Lucchi, R. Volpicelli, L. Cotarca, Top. Curr. Chem. 2007, 275, 131.

[16] G. C. Lloyd-Jones, J. D. Moseley, J. S. Renny, Synthesis 2008, 661.

[17] M. Roth, R. Pfaendner, P. Nesvadba, M.-O.Zink, WO Patent Publication No. WO2001090113, 2001.

[18] W. G. Skene, S. T. Belt, T. J. Connolly, P. Hahn, J. C. Scaiano, Macromolecules 1998, 31, 9103.

[19] The supplementary crystallographic data for 9 can be obtained free of charge from the Cambridge Crystallographic Data Centre via www.ccdc.cam.ac.uk/data_request/cif (Code CCDC-290324)

[20] E. R. Johnson, O. J. Clarkin, G. A. DiLabio, J. Phys. Chem. A 2003, 107, 9953.

[21] A. P. Scott, L. Radom, J. Phys. Chem. 1996, $100,16502$.

[22] F. Haase, R. Wolffenstein, Ber. Dtsch. Chem. Ges. 1904, 37, 3228.

[23] D. Charmot, H.-T. Chang, V. Nava-Salgado, US Patent Publication Application No. US 2004073042, 2004.

[24] F. M. Benitez, J. R. Grunwell, J. Org. Chem. 1978, 43, 2914.

[25] B. Maillard, K. U. Ingold, J. Am. Chem. Soc. 1976, $98,520$.

[26] N. Bricklebank, A. Pryke, J. Chem. Soc., Perkin Trans. 1 2002, 2048. 